\title{
Electroencephalogram Authentication Access Control to Smart Car
}

\author{
Yuhua Chen, Jinghai Yin
}

\begin{abstract}
In recent years, with the development of intelligent vehicles, the demand for security will be more and more big. One of the most important solutions is the use of new biometric technology. This paper presents an identity authentication system based on electroencephalogram (EEG) signals. The overall goal of this research is to design a new authentication method and develop the corresponding application. Therefore, we carried out a series of EEG experiments, and analyzed and discussed the experimental results. Based on these results, we study the algorithm of recognizing EEG signal features. Depended on the uniqueness of their EEG signals to be capable of authenticating access control to car, we build and present an Access Control System. The accuracy of authentication system is more than $87.3 \%$.
\end{abstract}

Keywords-Biometrics, Electroencephalogram, Smart Car, SVM, Fuzzy entropy.

\section{INTRODUCTION}

$\mathrm{A}$ uthentication system of smart car is an urgent part to be installed within smart car's compounds, which focused on security aspects of entry access. Conventional authentication systems use key and password, however, they are deemed inconvenience and insecurity. Biometrics appears to solve these problems related to conventional system. Currently, several relatively mature biometric technologies including fingerprint, face, iris, signature and speech recognition are well studied. These methods have its disadvantages and advantages depending on the performance, usability and capability. Therefore, there is a need to explore and find another method for biometric system. Electroencephalogram (EEG) signal is a very good biometric feature, which can be used as person authentication. As a potential source of identification technology, EEG has attracted extensive attention of researchers in recent years. It is stable enough for biometric analysis. At the same time, it can be easily recorded for a long time to obtain enough data for classifier training. Because of the difference of morphology and function, the brain needs to organize and coordinate the specific cognitive function or mental state. Recent studies have shown that some of the

This work was supported in part by 61762045, GJJ171030, 20181BBE50018, 16XTKFYB06.

Yuhua Chen is with the Center of Collaboration and Innovation, Jiangxi University of Technology, Yao Lake University Park, Nanchang 330098, China.

Jinghai Yin is with the Center of Collaboration and Innovation, Jiangxi University of Technology, Yao Lake University Park, Nanchang 330098 , China. (corresponding author; e-mail: peteryin1977@hotmail.com) specific information in EEG can be used to automatically identify the subject's identity. Due to the very high universality of EEG signal, and it also has a strong ability to resist deception, EEG is very suitable for identification as a biometric signal. Because it is impossible for an attacker to imitate an EEG signal of the tested person and transmit it to the corresponding electrode. When the subjects were collected in the resting state, the brain waves produced by brain activity were mainly distributed in the 0.5 to $40 \mathrm{~Hz}$ frequency band. There are five main types of rhythms that can be found in EEG signals. It is assumed that the brain is the slowest in the inactive state, while the fastest rhythm indicates that the brain is currently processing information. Different types of EEG signals can be decomposed by different mathematical methods. EEG signals are usually composed of many different waveforms. We can classify them according to their basic characteristics such as frequency, amplitude, waveform and spatial distribution.

EEG has been used in many different fields for clinical diagnosis. For example, verification of brain death or coma, identification of epileptic seizures, movement disorders, migraine, or deep anesthesia testing, etc.. Each person's EEG is as unique as his fingerprints. It has a very strong uniqueness. When a person receives visual stimulation, the visual cortex of the brain on the back of the head produces a strong change in the EEG signal. Therefore, this region is the best position to measure the visual EEG signal. In 2002,Palaniappan used Visual Evoked Potentials (VEPs) for biometric identification. The system extracted the Absolute Spectral Power(ASP) of the gamma band. They used 61 electrodes to collect EEG signals and then classified them using neural networks to get ASP features, achieving on about $90.95 \%$ of accuracy on ten subjects. Based on Mel frequency Cepstral coefficient (MFCC), energy and tunnel measurements, zero-crossing rate, spectral characteristics and their statistical functions, Nguyen et al. established a corresponding eigenvector model. The model achieved $92.8 \%$ of classification accuracies on 20 subjects, but the classification accuracies was about $61.7 \%$ on 122 subjects. Marcel and Millan developed a system, which including the imagined task of producing words, moving the right and the left hand. The system was based on Power Spectral Density (PSD) features. In the left hand moving task they obtain the best Equal Error Rate (EER) is $6.60 \%$. Thorpe et al. presented their idea for one type of authentication system by pass-thoughts and described the design of this system. Because the human brain signal is unique, when we carry out the same thinking activities, 
different people produce EEG signals are completely different. So they think such a certification system is feasible. RamKumar and Singhal have designed a system that can measure VEP by a single electrode. The classification accuracy of this system achieved $78 \%$ on 10 subjects. Das et al. studied the spatiotemporal pattern responsible for encoding personal discriminant data. The system achieved performances more that $90 \%$ for 20 subjects by a Linear Support Vector Machine (LSVM). Ferreira et al. obtained $84.33 \%$ accuracy in 13 subjects using support vector machine (SVM) based techniques Sun et al. put forward a multi-task learning method. They trained the neural network using the characteristics of multitask EEG signals, obtaining $95.6 \%$ and $94.81 \%$ of classification on imagine left and right hand moving task respectively. Su et al. used $5-32 \mathrm{~Hz}$ PSD as EEG characteristics and AR coefficients, and classified by combining LDC and $\mathrm{KNN}$, the system accuracy reached $97.5 \%$., when classifying with an $\mathrm{NN}$ and an SVM the system achieved $81.90 \%$ and $79.6 \%$ of accuracy. In the work of Bao et al., They use self-linear complexity, regression model, energy spectral density and phase synchronization as EEG features, and use neural networks to identify individual differences. Our previous research is mainly based on the personal identification of EEG signals, and the classification method based on multi feature fusion.

Some studies have shown that mobile devices can be used to collect EEG signals and send them to remote servers after signal pre-processing. Zúqueteet al. process VEP for the purpose of authentication and now use the image of the white and black line map by Snodgrass and Vanderwart to do an EEG authentication system. This is similar to the way we use it because we also use visual stimuli to create an identity authentication system. On the management of system security, we must first distinguish between three completely different concepts: identification, authentication and authorization. The main content of this paper is the system authentication. As a non-invasive BCI application, the EEG based biometric recognition system has received more and more attention in the scientific community. The identification of EEG signals is a relatively new method of biometric identification, which has benefited from the development of human genetics and clinical neurophysiology.

\section{METHOD}

The design of the new key for implementing the Access Control System (ACS) is developed using a model that actually works with various parts. These parts are summarized as shown in Fig. 1.Users through the Interface of ACS to control the authentication application software. Software directly call BCI middleware to achieve the core functions. The user's EEG signals are also sent to the middleware through the electrode cap. The middleware also exchange data with the algorithm library and the pattern library.

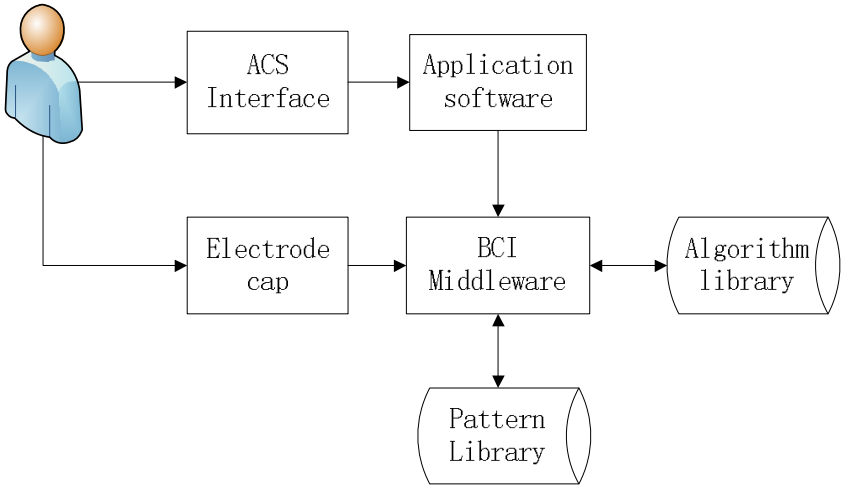

Fig. 1. Conceptual Components for ACS

\section{A. EEG Acquisition}

On the basis of previous research, we use 40-channel neuroscan amplifier to collect EEG signals, and use SCAN 4.3 software to record and analyze EEG data. According to the International 10-20 System (Fig. 2), two electrodes connected to the mastoid at A1 and A2 serve as reference electrodes for all other electrodes. The system uses 32-channel electrode caps (including 2 reference channels and 30 data channels) for signal acquisition. The sampling frequency of the system is set to $1000 \mathrm{~Hz}$, the filtering parameters are set to $200 \mathrm{~Hz}$ low pass and $0.05 \mathrm{~Hz}$ high pass.

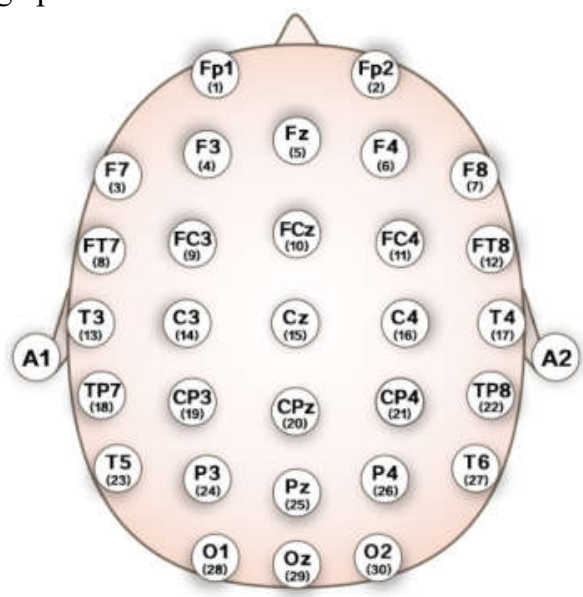

Fig. 2. Electrode Distribution Map Designated by International Standard

\section{B. Scenario}

The experiments are carried out in Collaborative Innovation Center of Automobile Service Engineering and Industrial Upgrading, Jiangxi University of Technology. Subjects are students from Jiangxi University of Technology. In the tests, subjects look towards display and perform operations according to the requirements of experiment design.

In the tests, stimulation program will display different pictures on computer screen. For each subject, five different pictures are displayed in each experiment. Each picture is randomly displayed on the screen for $1000 \mathrm{~ms}$, which is followed by a $250 \mathrm{~ms}$ black screen, totally taking $1250 \mathrm{~ms}$. For the five pictures displayed to the subject, there are one picture 
of the subject himself/herself and four background pictures. Each picture uses head part of subject with the same background.

\section{Data analysis}

The main process of feature extraction in this system includes establishing different types of data sets and calculating fuzzy entropy, the analysis of features and the calculation of Fisher distance. We performed a signal analysis of the two types of EEG data from both self and non self photographed responses. In order to realize the identification of human identity, we calculate the fuzzy entropy of these EEG signal. Its calculation formula is as follows:

Based on the time series of EEG data, $\{a(i): i \in[1, \cdots, N]\}$, we can reconstruct an $\mathrm{M}$-dimensional vector as shown below:

$$
\mathrm{U}_{\mathrm{i}}^{m}=\{\mathrm{u}(\mathrm{i}) \mathrm{\mu}(\mathrm{i}+1), \ldots \mathrm{\mu}(\mathrm{i}+\mathrm{m}-1)\}=\{a(i), a(i+1), \ldots, a(i+m-1)\}-a_{0}(i)
$$$$
(\mathrm{i}=1, \ldots, \mathrm{N}-\mathrm{m})
$$

where $\{\mathrm{u}(\mathrm{i}), \mathrm{u}(\mathrm{i}+1), \ldots, \mathrm{u}(\mathrm{i}+\mathrm{m}-1)\}, \quad\{a(i), a(i+1), \ldots, a(i+m-1)\}$ represent the continuous value of point $\mathrm{i}$, and $a_{0}(i)$ represents the average value of $\mathrm{m}$. The maximum distance can be generated by reconstructing the vector, and we can define it as follow:

$$
d_{i j}^{m}=d\left[U_{i}^{m}, U_{j}^{m}\right]=\max \{|u(i+k)-u(j+k)|\} k \in(0, m-1), \quad i \neq j
$$

and then the similarity degree, $\mathrm{D}_{i j}^{m}$, of $\mathrm{U}_{i}^{m}, U_{j}^{m}$ can be defined as follow:

$$
\left.\mathrm{D}_{\mathrm{ij}}^{m}=\mu\left(d_{i j}^{m}, n, r\right)=\exp \left(d_{i j}^{m}\right)^{n} / r\right)
$$

$\mathrm{R}$ and $\mathrm{N}$ are exponential functions of gradient and width. In this paper $\mathrm{R}=\mathrm{SD} * 0.3$ and $\mathrm{N}=2$. Therefore, we can define the following functions:

$$
\phi^{\mathrm{m}}(n, r)=\frac{1}{N-m} \sum_{i=1}^{N-m}\left(\frac{1}{N-m-1} \sum_{j=1}^{N-m} D_{i j}^{m}\right)
$$

When the fuzzy entropy of time series $\mathrm{N}$ is finite, its sequence length can be defined as:

$$
\text { FuzzyEn }=\left[\ln \varphi^{m}(n, r)-\ln \varphi^{m+1}(n, r)\right]
$$

We use Fisher distance method to analyze the characteristics of EEG signals collected by different electrodes. In classification research, Fisher distance is often used to represent the difference between classes. It is proportional to the distance between classes. The mathematical formula for calculating Fisher distance is as follows:

$$
F=\frac{\left(\mu_{1}-\mu_{2}\right)^{2}}{\sigma_{1}^{2}+\sigma_{2}^{2}}
$$

Among them, $F$ represents Fisher distance, $\sigma$ and $\mu$ represent variance and mean, subscripts 1 and 2 represent two types.

In order to provide an intuitive and easy-to-understand measurement method, this paper uses the following equations to test its performance.:

$$
\left\{\begin{array}{l}
F R R=\frac{F N}{T P+F N} \\
F A R=\frac{F P}{F P+T N}
\end{array}\right.
$$

In Formula 7, TP is true position, $\mathrm{TN}$ is true negative, $\mathrm{FP}$ is false positive, $\mathrm{FN}$ is false negative.

TP represents the number of EEG signals which is identified as a self portrait photograph. TN represents the number for non self portrait photographs that are identified as non self portrait photographs ofEEG. FP is the number that are identified as self time photos of EEG signals of non self portrait. FN is the number of the self portrait EEG signal is identified as non self portrait photos. FRR is the false rejection rate, and FAR is the false acceptance rate.

\section{Technical Front-end Setup}

The core modules of the control system are encapsulated in the middleware, which shields a large number of technical details, so that non professional developers can develop a variety of application software based on EEG. The system middleware architecture diagram is shown in Fig. 3, including the Android operating system, the device abstraction layer (Bluetooth interface, Wi-Fi interface, ZigBee interface, etc), the data management layer (containing some basic data operations such as data storage, data query, and data reception and so on), and some entities such as database, model library, algorithms library, EEG library and so on. In the core service layer, where all of the analysis and processing of EEG signals is completed, including the signal preprocessing, feature extraction and feature classification and other aspects of the complex process. The upper layer is the application interface, these applications include identity EEG, BCI, detection EEG, polygraph EEG, and so on. Each layer is called a layer of content, also a call, end users only need to call the application interface to complete the development work, without understanding how the EEG signal acquisition, processing and classification process is.

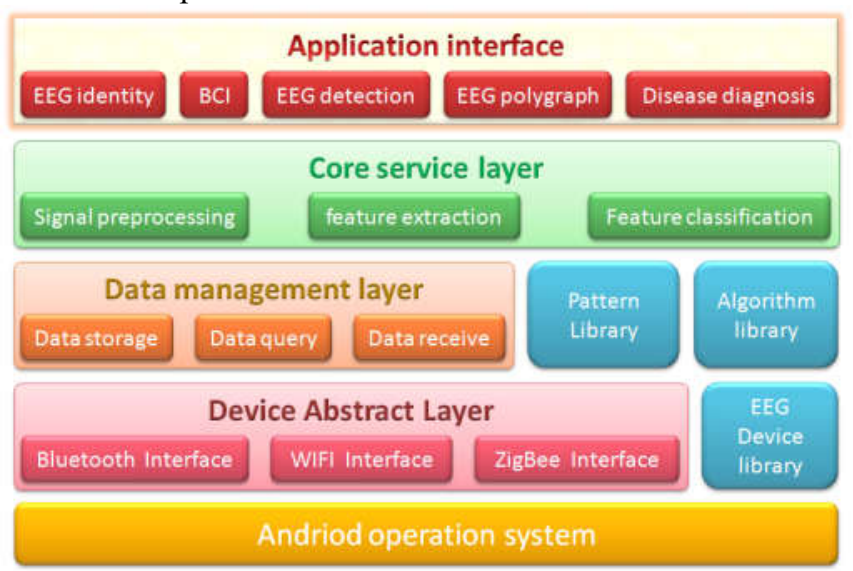

Fig. 3. Middleware architecture based on Android platform

Before recording the user's EEG signals to authenticate them, a series of steps need to be performed. From the user's point of view, the flow of the whole system is shown in Figure 4. After the EEG is collected through the signal preprocessing, feature extraction and other steps, system will produce a series of characteristic values. If the current operation type is the feature collection, these features will be saved to the feature database through several procedures, such as feature selection, feature classification, feature storage and so on. Once the 
identity of the operating system begin to match the characteristics and features of library data. If the match is successful, the engine can be started, otherwise system display "login failed", then system restart the identification process. If failed five times, the system access will be denied.

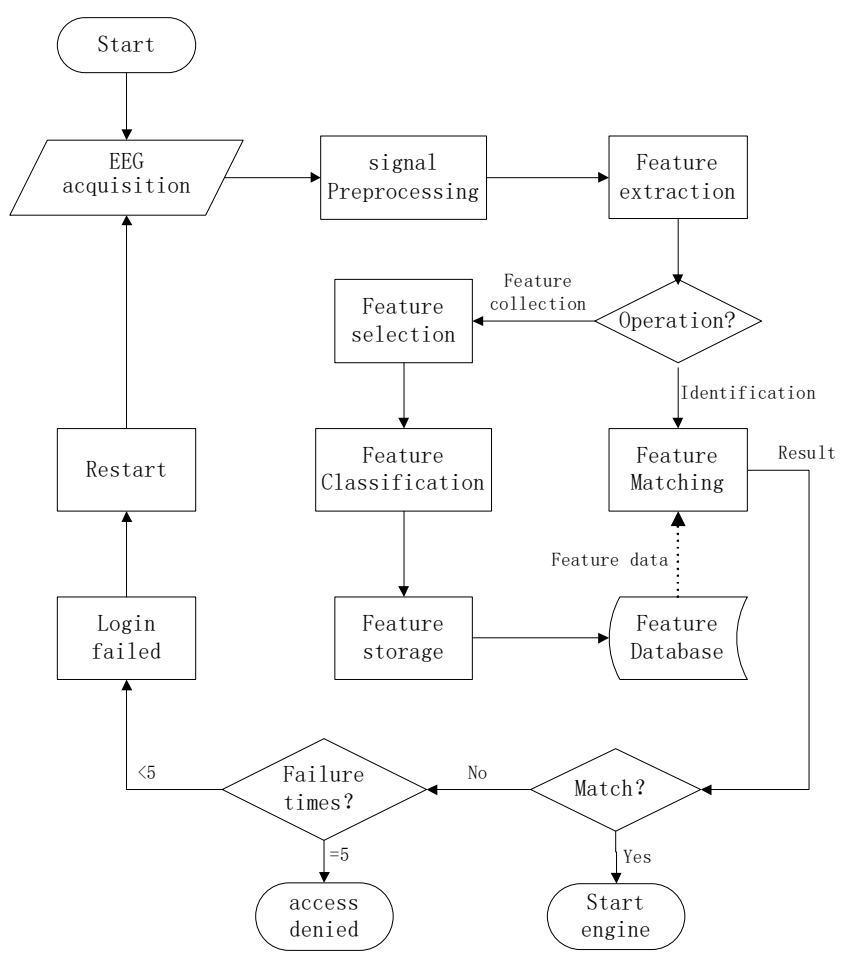

Fig. 4. System flow chart

\section{E. System structure}

The main function of the system is realized by the vehicle mounted mobile device.

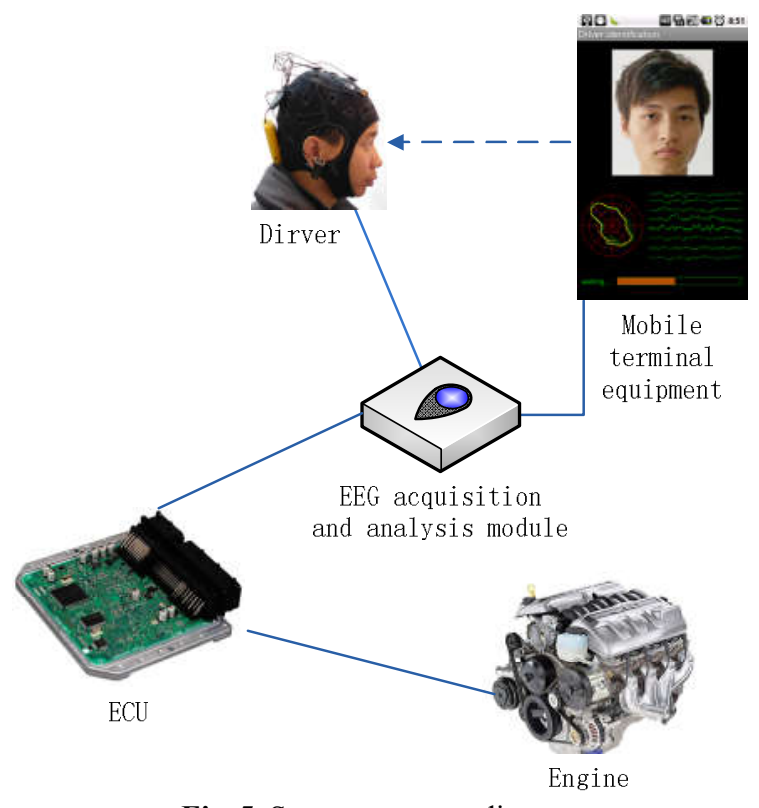

Fig. 5. System structure diagram

As shown in Fig. 5, the system is composed of Driver,
Mobile terminal equipment, EEG acquisition and analysis module, ECU and Engine. Mobile terminal devices will show the real time EEG signals and stimulation photos. The EEG signals of drivers are evoked by the photos stimuli, EEG signals are collected and analyzed after being sent to the mobile terminal equipment and ECU, ECU will identify the results into a signal to control the start of the engine.

\section{RESULTS}

As can be seen from table 1, we use our proposed identification method to identify EEG data, the average success rate in the electrode FP1 and FP2 fuzzy entropy is about $87.3 \%$. The highest classification accuracy was about $92 \%$ on 10 subjects, while the lowest was about $84 \%$. The method has a slightly lower error acceptance rate (FAR) 5.5\% and a lower false rejection rate (FRR) of 5.6\%. In addition, the average FAR and FRR of this method are relatively low. This shows that our proposed recognition method has good stability. The remarkable improved success rate indicates that using fuzzy entropy in the electrode FP1 and FP2 to design identify authentication access control is feasible.

Table 1. Performance of the authentication access system

\begin{tabular}{cccc}
\hline Subjects & Accuracy & FAR & FRR \\
\hline $\mathbf{1}$ & $85.4 \%$ & $8.0 \%$ & $6.4 \%$ \\
$\mathbf{2}$ & $90.0 \%$ & $6.6 \%$ & $3.3 \%$ \\
\hline $\mathbf{3}$ & $84.6 \%$ & $3.8 \%$ & $5.7 \%$ \\
\hline $\mathbf{5}$ & $92.1 \%$ & $3.1 \%$ & $4.6 \%$ \\
\hline $\mathbf{6}$ & $84.0 \%$ & $6.8 \%$ & $9.0 \%$ \\
\hline $\mathbf{7}$ & $87.3 \%$ & $6.2 \%$ & $3.1 \%$ \\
\hline $\mathbf{8}$ & $86.5 \%$ & $5.7 \%$ & $7.6 \%$ \\
\hline $\mathbf{9}$ & $90.7 \%$ & $3.6 \%$ & $2.4 \%$ \\
\hline $\mathbf{1 0}$ & $88.2 \%$ & $4.5 \%$ & $5.2 \%$ \\
\hline Mean (Std) & $87.3 \%(2.9)$ & $5.5 \%(1.7)$ & $5.6 \%(2.4)$ \\
\hline
\end{tabular}

It can be seen from Fig. 6 that there are two major data entities in this E-R diagram, the first entity is EEG features, the second entity is Feature vector, between both of them is $1: \mathrm{N}$ relationship, a feature entity corresponding to $\mathrm{N}$ eigenvectors. EEG features data entity includes Subject, Experimental Mode, Algorithm and Acquisition mode, and other related attributes. Feature vector data entity includes Vector matrix, Feature value, Number and other attributes. According to this ER diagram can 
infer basic data framework EEG database.

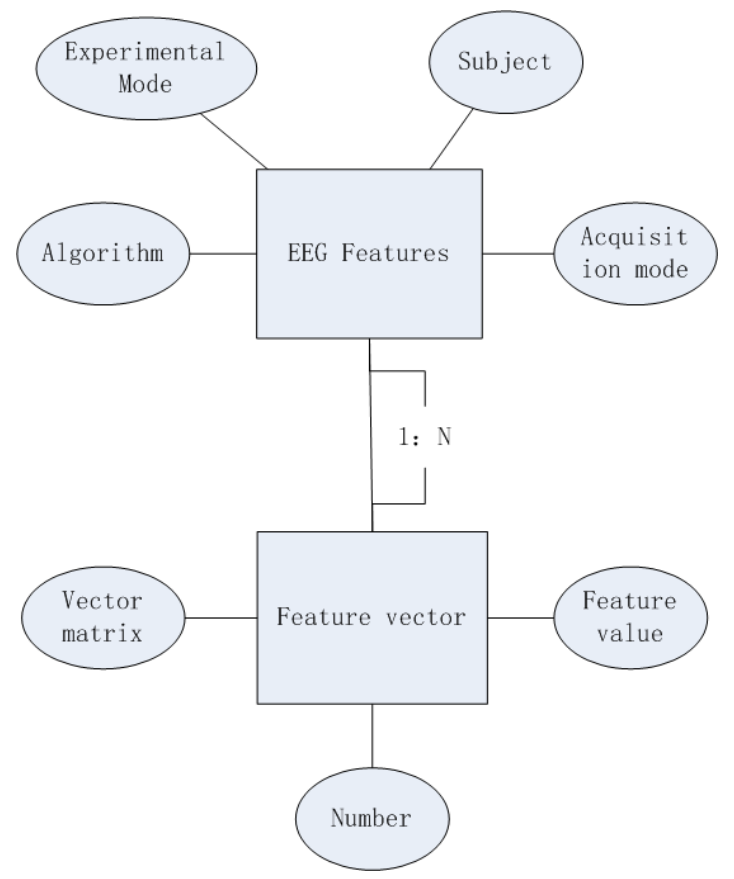

Fig. 6. Feature library E-R diagram

Fig.7 show the UI of driver's identity recognition program, the upper part of UI is the stimulation photos to induce specific EEG signals, the lower part is the change of EEG signals. And the progress bar is the time of single recognition tips.

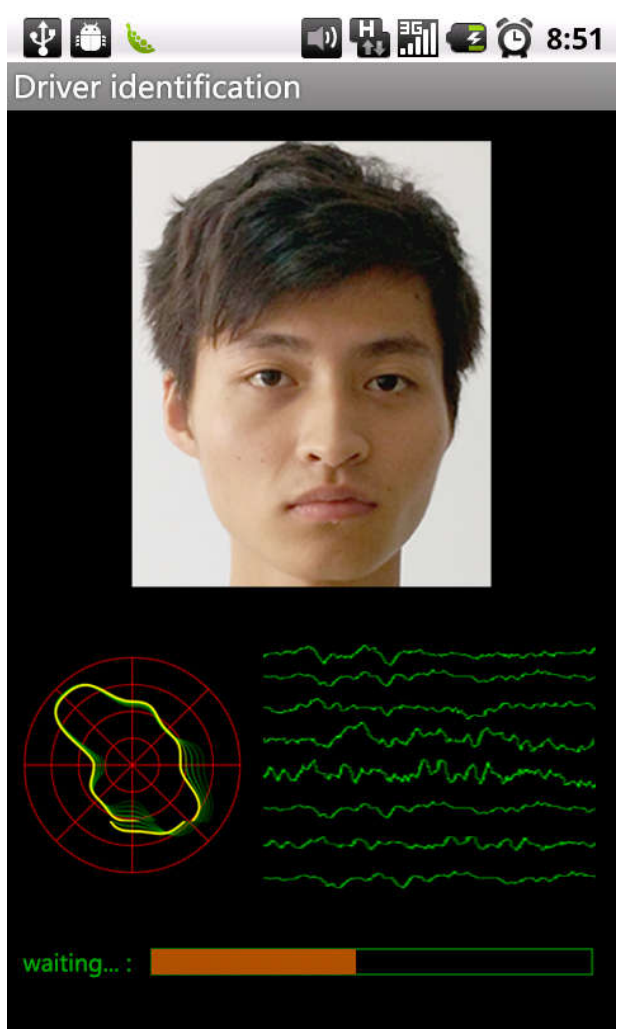

Fig. 7. Driver identification UI

\section{DisCUSSION AND CONCLUSION}

At the point, one can confirm that the problem of identity authentication ACS based on EEG signal is sensitive to some factors. Such as frequencies, the location of electrodes, stimulation paradigm and subject condition. Space and frequency may be two decisive factors of the problem. It seems clear that the spectral distribution of EEG signals contains subject specific features which can be used in an authentication system. These biometric systems can extract several characteristic behaviors. We can expect that multiple electrodes, rhythms and mission data can be used to increase the system's recognition performance.

The main disadvantage of EEG based personal identification is that the acquisition equipment is inconvenient to use. Nevertheless, many researchers are now working to design a variety of new EEG recording devices for users.

At present, people are trying to use some EEG based systems in our daily life. The problem of mobile acquisition of brain computer interface system based on single channel EEG signal has been solved. Using the mobile client as the foundation of the system platform, a simple EEG based authentication system can be established by using a low-cost acquisition device. The monitoring of the impedance of the dry electrode and the integrated skin to electrode as well as the reliable high quality wearable EEG monitoring system have also been developing continuously. With the rapid development of these technologies, the acquisition equipment becomes more and more practical and reasonable. We believe that in the near future there will be cheap and practical EEG based authentication system.

\section{REFERENCES}

[1] M. Merone, P. Soda, M. Sansone, and C. Sansone, "ECG databases for biometric systems: a systematic review", Expert Systems with Applications, vol. 67, no.8, pp. 189-202, 2017.

[2] Z. Yao, J. Bars, L. M., C. Charrier, and C. Rosenberger, "Literature review of fingerprint quality assessment and its evaluation", IET Biometrics, vol. 5, no.3, pp. 243-251, 2016.

[3] X. Zhang, D. Pham, S. Venkatesh, et al. "Mixed-norm sparse representation for multi view face recognition", Pattern Recognition, vol. 48, no.9, pp. 2935-2946, 2015.

[4] D. Miao, M. Zhang, Z. Sun, T. Tan, and Z. He, "Bin-based classifier fusion of iris and face biometrics", Neurocomputing, vol. 224, no.5, pp. 105-118. , 2017.

[5] F. J. Zareen, and S. Jabin, "Authentic mobile-biometric signature verification system", IET Biometrics, vol. 5, no.1, pp. 13-19, 2016.

[6] G. Bajwa, and R. Dantu, "Neurokey: Towards a new paradigm of cancelable biometrics-based key generation using electroencephalograms", Computers \& Security, vol. 62, no.7 pp. 95-113, 2016.

[7] D. Rodrigues, G. Silva, J. Papa. et al. "EEG-based person identification through Binary Flower Pollination Algorithm", Expert Systems With Applications, vol. 62, no.2, pp. 81-90, 2016.

[8] E. Maiorana, D. La Rocca, P. Campis, "Eigenbrains and Eigentensorbrains: Parsimonious bases for EEG biometrics", Neurocomputing, vol. 171, no.3, pp. 638-648, 2016.

[9] E. Maiorana, J. Solé-Casals, and P. Campisi, "EEG signal preprocessing for biometric recognition", Machine Vision \& Applications, vol. 27, no.8, pp. $1-10,2016$ 
[10] JH. Yin, JF. Hu, ZD. Mu, "Developing and evaluating a Mobile Driver Fatigue Detection Network Based on Electroencephalograph Signals", Healthcare Technology Letters, vol. 4, no.1, pp. 34-38, 2017

[11] ZD. Mu, JF. Hu, JH. Yin, "Driving Fatigue Detecting Based on EEG Signals of Forehead Area", International Journal of Pattern Recognition and Artificial Intelligence. vol. 31, no.5, pp. 175-186, 2017

Yuhua Chen was born on Oct. 4, 1976. She received her masters degree in Computer Software and Theory from Nanchang University in 2010. She is currently a lecturer at Jiangxi University of Technology. Her research interests are mainly in artificial intelligence.

Jinghai Yin was born on Jan. 28, 1977. He received his masters degree in Computer Software and Theory from Nanchang University in 2005 . He is currently a Professor at Jiangxi University of Technology. His research interests are mainly in brain computer interface and artificial intelligence.

\section{Creative Commons Attribution License 4.0 (Attribution 4.0 International, CC BY 4.0)}

This article is published under the terms of the Creative Commons Attribution License 4.0

https://creativecommons.org/licenses/by/4.0/deed.en_US 